\title{
EFECTOS DEL PARASITISMO SOBRE EL TEJIDO BRANQUIAL EN PECES CULTIVADOS: ESTUDIO PARASITOLOGICO E HISTOPATOLOGICO
}

\author{
Maurício Laterça Martins ${ }^{1}$ \\ Nicolás Garcia Romero ${ }^{2}$
}

\begin{abstract}
EFFECT OF THE PARASITISM ON THE GILL TISSUE OF CULTIVATED FISHES: PARASITOLOGICAL AND HISTOPATHOLOGICAL STUDIES. One hundred four out of 225 diagnosticated cases were myxosporidian, monogenean, Ichthyophthirius multifiliis Fouquet, 1876 and bacterial diseases in Piaractus mesopotamicus Holmberg, 1887 (pacu), Colossoma macropomum Cuvier, 1818 (tambaqui) and tambacu, at Aquaculture Center, Universidade Estadual Paulista (UNESP), Jaboticabal, São Paulo, Brazil, between 1992 and 1995 . The gills were fixed in $10 \%$ buffered formalin solution to posterior histologic routine. It was observed Henneguya sp. cysts into the capillaries of the secondary lamellae, encapsulated by the respiratory epithelium cells. It provoked adherence of the adjacent lamellae, hyperplasia, congestion, oedema and epithelium displacement. Monogeneans infestations by Anacanthorus penilabiatus Boeger, Husak \& Martins, 1995 and Ancyrocephalinae sub-family caused light inflammatory reaction and hyperplasia. In severe infestations was observed hyperplasia of primary lamellae, necrosis, oedema, respiratory epithelium displacement, ruptured pillar cells and telangiectasis. Response to $I$. multifiliis was limited to surrounding epithelial cells in young fishes and hyperplasia, necrosis, inflammatory infiltrate and oedema in old fishes. Such lesions iniciate hyperplasic and oedematous process that with inflammation of the parasitic sites, provoked alterations over gases and ions interchange surface and consequently fish metabolism.
\end{abstract}

KEY WORDS. Protozoa, Monogenea, histopathology, Brazilian fish

La expansión de la piscicultura de agua dulce en Brasil, ha comenzado a prestar especial atención a las especies nativas, muchas de las cuales no están adaptadas al cautiverio. Este hecho genera incomvenientes, ya que, dado el incipiente desarrollo de estos cultivos, no se conoce con certeza los requerimientos nutricionales, las densidades de cultivo y el manejo más adecuado de peces e instalaciones. Consecuentemente, comienzan a surgir problemas nutricionales y de enfermedades infecciosas y parasitarias. Es ampliamente conocida la estrecha relación que existe entre hospedador-patógeno - ambiente, marcando un delicado equilibrio que, si es quebrado, sobreviene la enfermedad (KINKELIN et al. 1991). De acuerdo con ROBERTS \& BULLOCK (1980) existen parásitos y bacterias que pueden estar normalmente presentes en la superficie del cuerpo y branquias de los

1) Centro de Aqüicultura, Universidade Estadual Paulista. Rodovia Carlos Tonanni Km 05, 14870-000 Jaboticabal, São Paulo, Brasil.

2) Sección de Ictiología, Universidad Nacional de La Plata. 1900 La Plata, Argentina. 
peces y, sólo en condiciones favorables para ellos, proliferan para tornarse patógenas.

Las alteraciones conductuales que provocan las parasitosis sobre los peces son muy semejantes. Entre ellas, podemos citar apatia, aglomeración en la superficie, entrada de agua y natación desordenada (CONROY 1987).

Poco ha sido hecho en relación a la histopatología de peces brasileros afectados por mixosporidios, Ichthyophthirius multifiliis Fouquet, 1876 y monogeneos, a pesar de ser estos parásitos los de más alta incidencia y los que causan mayor daño al los cultivos comerciales.

Las infecciones provocadas por Henneguya sp. (Myxozoa, Myxobolidae) causan patologias que pueden ser responsables de importantes mortandades en cultivos intensivos y semintensivos (DYKOVA \& LOM 1978; MCCRAREN et al. 1975; DUHAMEL et al. 1986; KALAVATI \& NARASIMHA-MURTI 1985; AgUiRRE et al. 1983; MARTINS et al. no prelo).

Ichthyophthirius multifiliis es un protozoario responsable de grandes pérdidas en el cultivo de peces, principalmente relacionado a operaciones de manejo en las épocas frías el año. HINES \& SPIRA (1974), VENTURA \& PAPERNA (1985) y EWING et al. (1985), describieron los procesos histopatologicos relacionados a esta parasitosis. LEIBOVITZ (1980a) y KUROVSKAYA \& OSADCHAYA (1993) observaron los efectos de la ictiftiriase en alevinos.

Otras parasitosis causales de problemas en el cultivo de peces en Brasil, son las infestaciones por monogeneos, responsables del incremento de las mortalidades por acción directa o por ser iniciadoras de otros procesos infeciosos como bacteriosis y micosis (Leibovitz 1980b; CeCCARELli et al. 1990; FigUeIRA \& CeCCARElli 1991). Los efectos causados por estos parásitos sobre la superficie del cuerpo y de las branquias fueron estudiados por CUSACK (1986) y BUCHMANN et al. (1987) respectivamente.

En el presente trabajo los autores descriven la ocurrencia e histopatologia de Henneguya sp., I. multifiliis y monogenea (Anacanthorus penilabiatus Boeger, Husak \& Martins, 1995 y Ancyrocephalinae) en peces analizados en el Centro de Acuicultura de la Universidade Estadual Paulista (UNESP), Jaboticabal, São Paulo, Brasil.

\section{MATERIAL Y METODOS}

Durante el período comprendido ente 1992 y 1995 fueron analizados y diagnosticados 225 casos de parasitosis, bacteriosis y micosis enviadas al Centro de Acuicultura del la UNESP, provenientes de todo el estado de Sao Paulo y algunos del estado de Paraná.

Los peces eran acondicionados en bolsas plásticas con un tercio de agua y completadas con oxígeno. Cada una contenía de 3 a 10 ejemplares, dando preferencia en la colecta a los individuos moribundos o con alguna señal de enfermedad.

El procediminento para el análisis parasitológico consistiá del raspado de la superficie del cuerpo y aletas para su posterior visualización en estereomicroscopio 
sobre placa de Petri, para luego completar las observaciones con microscopio óptico comun. Seguidamente, las branquias eran retiradas con material quirúrgico para ser visualizadas sobre una placa de Petri con solución fisiológica a la lupa binocular. Cada tipo de parásito requirió de la utilización de metodologias especificas de colecta y preparación (AMATO et al. 1991):

- Myxozoa. Al detectarse su presencia en los extendidos del exámen parasitológico de rutina, el portaobjetos era secado al ambiente, fijado con alcohol metílico, secado nuevamente y teñido con Giemsa (MARTINS et al. no prelo).

- Ichthyophthirius multifiliis. Cuando los raspajes realizados a aletas, superficie del cuepo y branquias contenían a estos protozoos, el portaobjeto era secado al ambiente, fijado en alcohol metílico y teñido con Giemsa (CONROY 1987).

- Monogenea. Los parásitos ubicados sobre la superficie del cuerpo fueron colectados, luego de sumergir el cuerpo del pez en formalina 1: 4000 y dejando descansar por dos horas. Las branquias siguieron igual procedimiento dejando reposar por dos horas y agitada de 20 a 40 veces para desprender los parásitos, fijados en formalina 5\%. Posteriormente, el material fue teñido con hematoxilina de Delafield o Carmin, diafanizados con creosota de Faia y visualizados al microscopio (AMATO et al. 1991).

Acompañando el estudio parasitológico se tomaron muestras de tejido branquial para su procesamiento histológico. En las muestras que fueron fijadas en formol buffer al 10\%, post fijación, se utilizó líquido de Bouin por tres horas. Este fijador fue utilizado, principalmente, para lograr el ablandamiento de las porciones osificadas de tejido branquial. Posteriormente, fueron deshidratados en concentraciones crecientes de alcohol etílico $\left(70^{\circ}, 90^{\circ}\right.$ y $\left.100^{\circ}\right)$ y luego, aclarados en xilol. Los tejidos se incluyeron en parafina, confeccionándose un taco por ejemplar. Los cortes se hicieron con un micrótomo con un espesor entre 5 y $7 \mu \mathrm{m}$. Las secciones histológicas fueron coloreadas con Hematoxilina - Eosina y observadas con el fotomicroscopio Wild.

\section{RESULTADOS}

De las 225 necropsias, 220 peces se encontraban parasitados (Tab. I). De ellos 111 fueron pacús (Piaractus mesopotamicus Holmberg, 1887), 68 tambaquís (Colossoma macropomum Cuvier, 1818) y 46 tambacús (híbridos de Colossoma macropomum y Piaractus mesopotamicus).

\section{OBSERVACIONES PARASITOLÓGICAS}

Myxozoa. Las branquias presentaban cistos de coloración oscura de 0,1 a $0,9 \mathrm{~mm}$ de diámetro dispersos entre las lamelas secundarias. La extremidad de los filamentos branquiales presentaban coloración "parda". El exámen microscópico de los cistos concluyó con la identificacón de esporas fusiformes de 48,4 $10,0 \mu \mathrm{m}$ de largo, con un apéndice caudal bifurcado y dos cápsulas polares en la extremidad anterior cada una con un filamento polar en cada cápsula, correspondientes a Henneguya sp. Thélohan, 1892 (Myxozoa, Myxobolidae) de acuerdo con MARTINS et al. (no prelo). 
Tabla I. Ocurrencia de patógenos entre 1992 y 1995 en el Centro de Acuicultura de la UNESP, Jaboticabal. São Paulo.

\begin{tabular}{|c|c|c|c|c|c|c|c|}
\hline \multirow{2}{*}{ Patogeno } & \multicolumn{2}{|c|}{ Colossoma macropomum } & \multicolumn{2}{|c|}{ Piaractus mesopotamicus } & \multicolumn{2}{|c|}{ Tambacu } & \multirow{2}{*}{ Total } \\
\hline & No. indiv. & $\%$ & No. indiv. & $\%$ & No. indiv. & $\%$ & \\
\hline Flexibacter columnaris & 6 & 8,8 & 5 & 4,5 & 2 & 4,3 & 13 \\
\hline Otras bacterias & 5 & 19,1 & 4 & 4,5 & 2 & 4,3 & 11 \\
\hline Saprolegnia sp. & 13 & 19,1 & 4 & 3,6 & 5 & 10,8 & 22 \\
\hline Ichthyophthirius multifiliis & 13 & 19,1 & 8 & 7,2 & 13 & 28,2 & 34 \\
\hline Trichodina sp. & 1 & 1,5 & 6 & 5,4 & 2 & 4,3 & 9 \\
\hline Chilodonella sp. & 1 & 1,5 & 1 & 0,9 & 1 & 2,2 & 3 \\
\hline Henneguya sp. & & & 17 & 15,3 & 1 & 2,2 & 18 \\
\hline Otros protozoos & 2 & 2,9 & 3 & 2,7 & 2 & 4,3 & 7 \\
\hline Monogenea & 13 & 19,1 & 37 & 33,3 & 8 & 17,4 & 58 \\
\hline Digenea & & & 3 & 2,7 & 1 & 2,2 & 4 \\
\hline Cestoidea & 1 & 1,5 & & & & & 1 \\
\hline Nematoda & & & 4 & 3,6 & 1 & 2,2 & 5 \\
\hline Argulus sp. & & & 2 & 1,8 & & & 2 \\
\hline Dolops sp. & 1 & 1,5 & & & & & 1 \\
\hline Lernaea cyprinacea & 9 & 13,5 & 15 & 13,5 & 6 & 13,0 & 30 \\
\hline Ergasilidae sp. & & & & & 1 & 2,2 & 1 \\
\hline Causas no determinadas & 3 & 4,4 & 1 & 0,9 & 1 & 2,2 & 5 \\
\hline TOTAL & 68 & & 111 & & 46 & & 225 \\
\hline
\end{tabular}

Monogenea. La observación de las branquias a la lupa binocular evidencio la presencia de gran cantidad de estos parásitos a lo largo de los filamentos branquiales anclados en las laminillas secundarias. Se destacaron puntos hemorrágicos y aumento en el volumen de los filamentos.

Se logró identificar a Anacanthorus penilabiatus Boeger, Husak \& Martins, 1995 presentes en $P$. mesopotamicus, midieron 560 a $894 \mu \mathrm{m}$ de largo, provisto de pequeños ganchos marginales en la extremidad posterior (opistaptor). En C. macropomum fueron hallados individuos pertenecientes a la sub-familia Ancyrocephalinae que midieron de 90 a $186 \mu \mathrm{m}$ de largo, provistos de dos pares de ganchos una de ellas bastante larga $(63,7 \pm 18,3 \mu \mathrm{m})$ y el otro menor $(27,1 \pm 9,3 \mu \mathrm{m})$ (PRIETO et al. 1994).

Ichthyophthirius multifiliis. Fouquet, 1876. Los peces presentaban los típicos puntos blancos en la superficie del cuerpo, aletas y branquias de $C$. macropomum y $P$. mesopotamicus. Trofontes de $0,2 \mathrm{a} 1 \mathrm{~mm}$ de diámetro, esféricos y de nucleo en forma tipica de herradura. También se hallaron terontes (formas infetantes) ovales y ciliadas de 30 a $45 \mu \mathrm{m}$.

\section{OBSERVACIONES HISTOPATOLÓgICAS}

Myxozoa. Los quistes de plasmodios se ubican en los capilares sanguineos de las laminillas secundarias. Son rodeados, primeramente, por endotelio hipertrófico. El plasmodio crece y queda encapsulado por células planas del epitelio respiratorio, mientras que el epitelio estratificado columnar tiende a desaparecer.

El crecimiento de quiste genera: adherencias entre las laminillas secundarias adyacentes, respuesta hiperplácica para encapsular al quiste (Fig. 1a), congestión lamelar y edema que lleva al desprendimiento del epitelio respiratorio (Fig. 1b). 

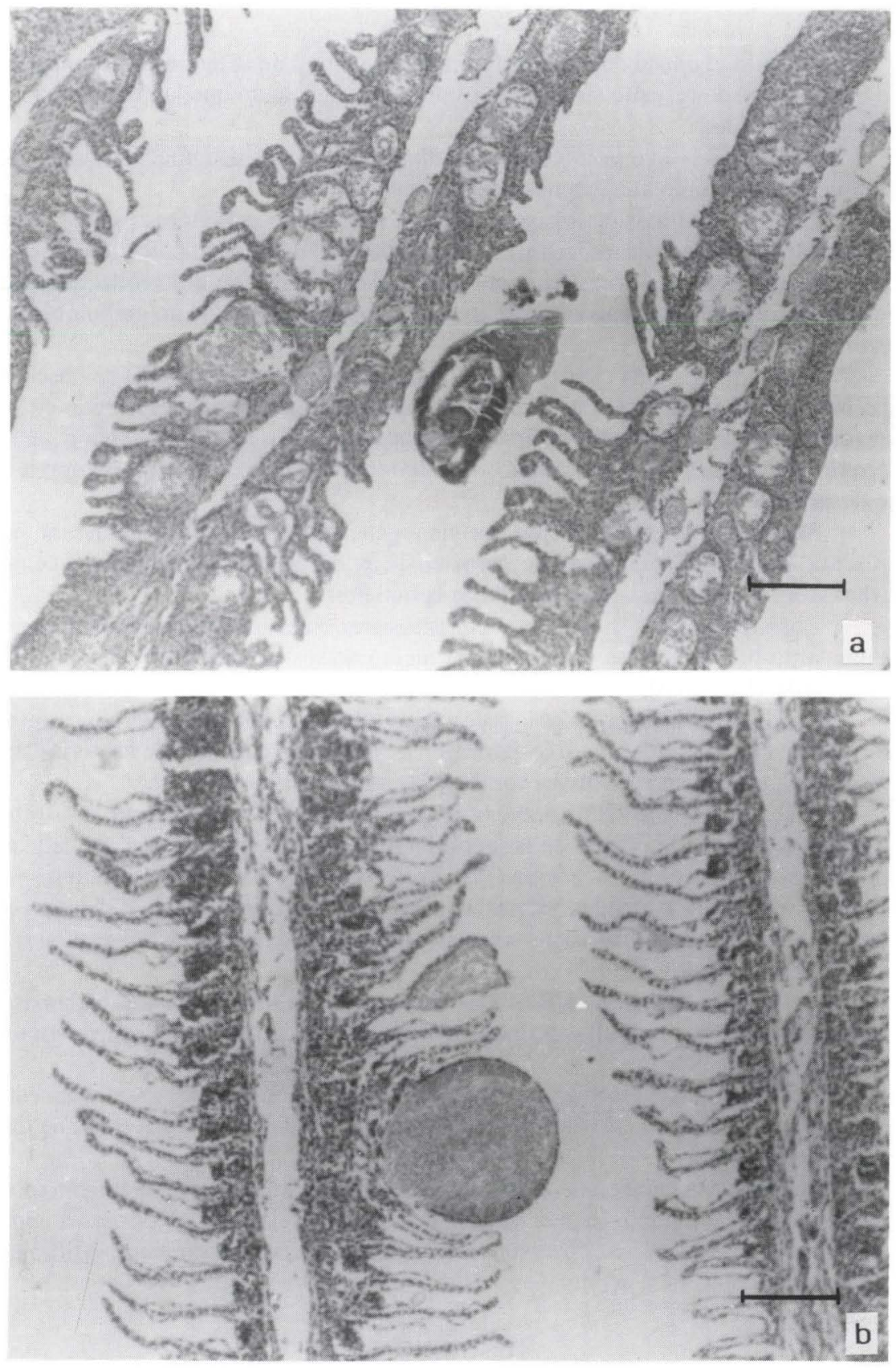

Fig. 1. (a) Quistes de Henneguya sp. en las laminillas secundarias con reaccion hiperplasica; (b) desprendimiento del epitelio respiratorio en $P$. mesopotamicus. Hematoxilina-Eosina, escala: $100 \mu \mathrm{m}$. 
Cuando el quiste ocupa la porción media y basal de la laminilla secundaria, se observó que la restante sección no es afectada, indicando que la circulación no fué interrumpida.

En los casos que la infección de mixosporidios fué acompañada por la de monogeneos, se halló una respuesta tisular incrementada.

En los individuos en los que se supone infecciones anteriores (mayor edad en una zona endémica de infección), la respuesta tisular es sumamente violenta con una reacción hiperplasica masiva para encapsular al quiste en desarrollo, seguida por la invasión de macrófagos y culminando con la formación de un granuloma de reconstrucción.

En los meses más calidos (temp. agua aproximadamente $26^{\circ} \mathrm{C}$ ) se observó una marcada sincronización en el estado de desarrollo de los quistes, siendo en su mayoria maduros o en las etapas finales de maduración; los meses frios (temp. agua aproximadamente $19^{\circ} \mathrm{C}$ ). En cambio, se hallaron más quistes en etapas tempranas de desarrollo.

Monogenea. La infestación por monogeneos en grado leve y moderado no presenta una respuesta tisular de características destacables, sólo una reacción inflamatoria e hiperplasia leve de células epiteliales.

Los monogeneos se aplican preferentemente en las porciones media y basal de las laminillas primarias, fijándose por medio de sus ganchos entre las lamelas secundarias (Fig. 2a,b).

En la mayoria de los casos la presencia de monogeneos se hallava acompañada por otros parásitos como mixosporidios (I. multifiliis) y quistes de bacterias sin diferenciar, siendo las reacciones combinadas más evidentes (Fig. 1a).

En casos de parasitismo grave (más de 10 monogeneos por campo de \# 100), se observa en la región distal de la laminilla primaria hiperplasia basal de celulas epiteliales, focos necróticos, edema (Fig. 3) con desprendimiento de epitelio respiratorio y rupturas de células pilares con fenómenos telangiectasicos basales y distales, a su vez se hallaron casos con rupturas del endotelio capilar con estravasación de eritrocitos.

Ichthyophthirius multifiliis. Los trofontes se hallaron adheridos en la base sector central de las laminillas primarias, estando recubiertos por una capa simple de celulas epiteliales.

Los protozoos presentaban restos de células semidigeridas y vacuolas con residuos celulares en su citoplasma indicadoras de la acción parasítica sobre de los tejidos del huesped.

La respuesta tisular a la acción del parásito se limitó al encapsulamiento por medio de células epiteliales en infecciones de peces jovenes y en casos mas avanzados de infestación se observaron focos hiperplasicos, necróticos e infiltrado inflamatorio con edema acompañante (Fig. 4).

\section{DISCUSION}

Las observaciones realizadas junto con la bibliografia consultada, nos permitió generar una tipificación de los distintos tipos de respuesta tisular a las parasitosis consideradas. 

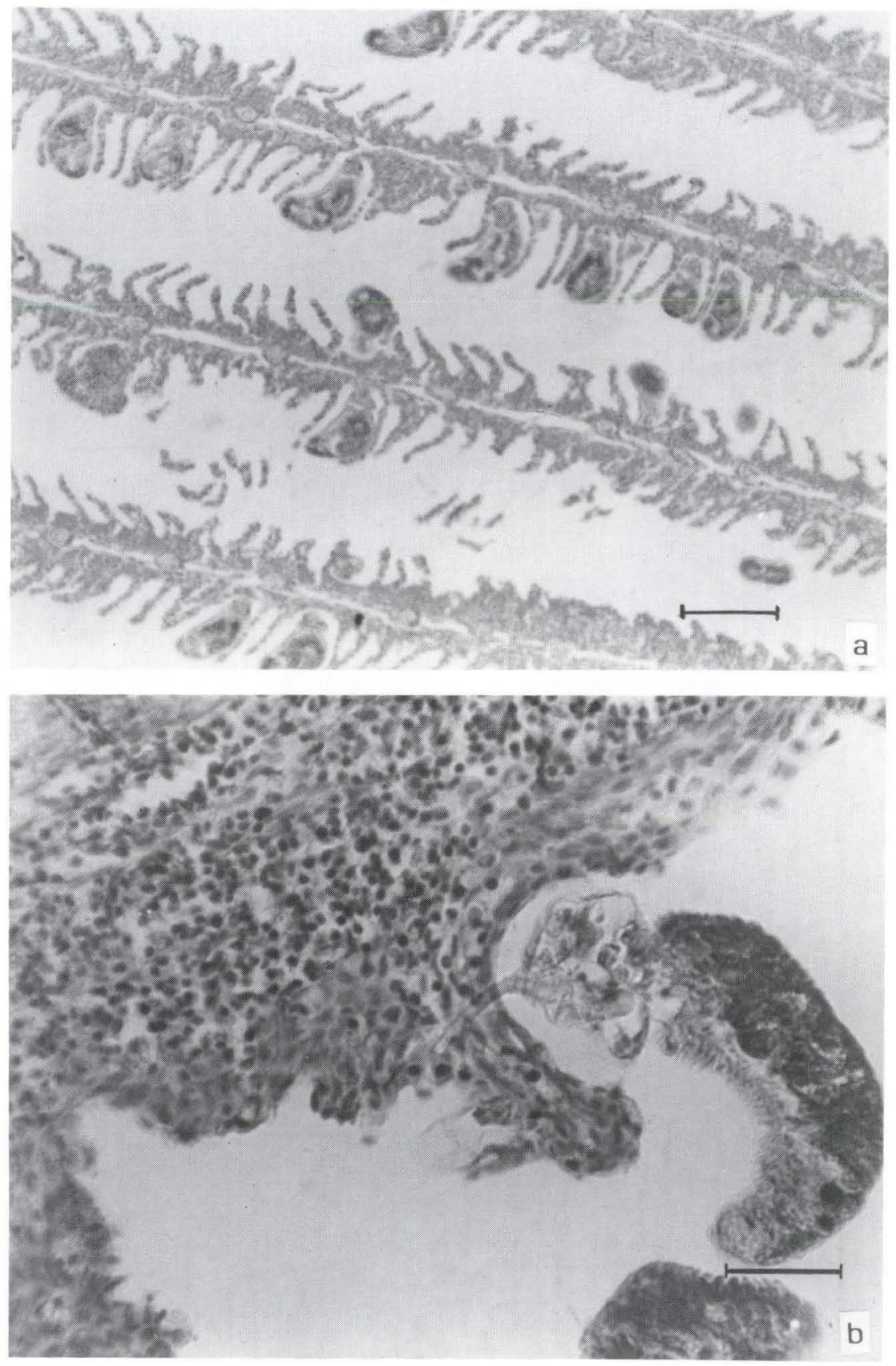

Fig. 2. Fijacion de monogeneos en el tejido branquial de $C$. macropomum. Hematoxilina-Eosina, (a) escala: $100 \mu \mathrm{m}$, (b) escala: $30 \mu \mathrm{m}$. 


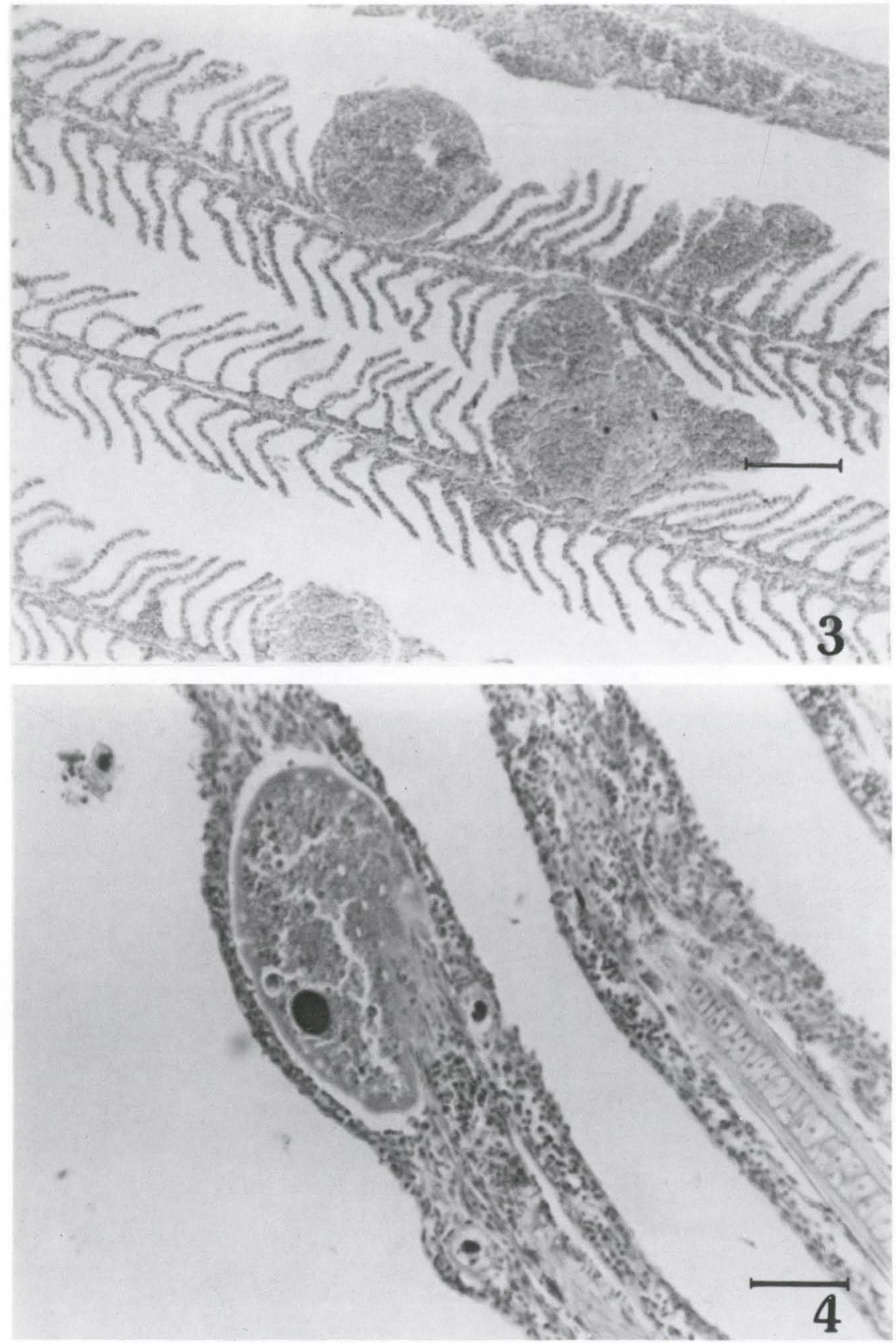

Figs 3-4. (3) Edema de la laminilla en caso de parasitismo grave por monogeneos en $C$. macropomum. Hematoxilina-Eosina, escala: $100 \mu \mathrm{m}$; (4) trofontes de I. multifiliis en las laminillas primarias de C. macropomum. Hematoxilina-Eosina, escala: $50 \mu \mathrm{m}$. 
Los hallazgos patologicos ante la infección por mixosporidios del género Henneguya sp., se hacían evidentes en la observación macroscopica del tejido branquial, presentando la extremidad de los fillamentos branquiales congestión sanguínea dando una coloración pardusca. El exámen histopatológico halló el sustento para las observaciones preliminares y mostró la presencia de diferencias estructurales según el estado de desarrollo de la infección quiste (DYKOVA \& LOM 1978). Primeramente, el quiste es rodeado con endotelio hipertrófico; seguidamente, el crecimiento del quiste genera hiperplasia, edema, citolisis e inflamación que van en incremento hasta la maduración de los mixosporidios.

También, el desarrollo de procesos granulomatosos de reparación (DYKOVA \& LoM 1978; DUHAMEL et al. 1986) fue observado en mayor grado en peces que poseían una alta probabilidad de haber sido infectados sucesivamente por ser individuos mayores del año que habitan una zona endémica de infección (CHENG 1973; ROBERTS 1981).

Esta última observación nos habla de la capacidad de "memoria" que poseen los huespedes hacia todas las parasitosis aquí descriptas (ELLIS et al. 1976). En todos los casos se halló una respuesta tisular incrementada con invasión de macrófagos y neutrofilos al granuloma en individuos de mayor edad (ANDERSON 1990). A su vez las mayores mortalidades atribuibles a I. multifiliis y Henneguya sp., se producen en larvas y juveniles de las especies consideradas (CECCARELLI et al. 1990; FigueIRA \& CECCARELli 1991), siendo éstas las más vulnerables por el escaso desarrollo de su sistema de defensa (HERRAEZ \& ZAPATA 1986; STOSKOPF 1993).

La tercera en importancia se hallo en las infecciones por $I$. multifiliis, observandose parasitismos agudos en juveniles de las especies muestreadas, con trofontes y terontes tanto en la superficie del cuerpo como en el tejido branquial. A pesar de la alta incidencia de protozoos presentes, la respuesta tisular fue sumamente leve, limitándose al encapsulamiento por parte de células epiteliales. Este panorama cambia radicalmente en peces de mayor edad, donde se hallan focos hiperplásicos, infiltrado inflamatorio y necrosis en las zonas más afectadas. Las observaciones coinciden con las realizadas por VENTURA \& PAPERNA (1985) para infecciones crónicas de peces de cultivo.

Por último, los monogeneos A. penilabiatus y los pertenecientes a la sub-familia Ancyrocephalinae hallados, no generaron conspicuas lesiones y por lo tanto no fue hallado un desarrollo defensivo de importancia en la zona de fijación de los parasitos. Llamativamente en casos de infeciones severas se halló una alteración notable de la porción distal de la laminilla primaria y las laminillas secundarias, asociadas con múltiples hemorragias, ederna con desprendimiento del epitelio respiratorio y evidentes focos necróticos. No hallamos otro agente asociado a la patologia que pudiera ser causal de la misma, sólo la alta incidencia de monogeneos.

No fueron hallados datos bibliográficos que describan igual situación por lo que se genera un importante antecedente para ser tomado en cuenta en futuros trabajos, ya que una lesion del tipo descripta y tan generalizada en el tejido branquial, conduce a alteraciones en el metabolismo general del pez de suma importancia. Todas las lesiones parasíticas descriptas coinciden en dos puntos de suma importancia, en mayor o menor grado todas son iniciadoras de procesos 
hiperplásicos y edematosos que alteran sustancialmente la superficie de intercambio gaseoso e iónico, generando un atendible desbalance osmótico y un grave problema en la oxigenación y equilibrio acido-base del pez. Complementariamente, los procesos inflamatorios descriptos para los sitios de fijación parasitica, en muchos casos generan patologia en si mismas, por la cronicidad del estímulo (ElLIS 1981; ROBERTS 1981; TORROELA 1988). Este trabajo muestra que existe una clara y única relación entre los procesos parasitarios y la respuesta tisular branquial que provocan alteraciones en los procesos normales de intercambio gaseoso y de iones. A su vez se halló una respuesta diferencial a los distintos parásitos y a las previas expociciones a los mismos. Sin embargo, parece haber una tendencia de acostumbramiento entre los hospedadores y los monogeneos y I. multifiliis, siendo en estos casos las menores respuestas tisulares. En cambio, para el caso de Henneguya sp., se observan cambio violentos aún en casos de infeciones en ejemplares jóvenes. La razón de estos hechos se podría encontrar en futuros trabajos que contemplen específicamente los procesos patológicos causados por parasitosis, en distintos estadios del parásito y del pez, sin olvidar las variables ambientales.

\section{REFERENCIAS BIBLIOGRAFICAS}

Aguirre, M.; D.A. Conroy; G. Conroy \& J.M. Torrealba. 1983. Black spot disease, a myxosporidiosis affecting silver mullet (Mugil curema Val.) in Venezuela. Riv. It. Piscic. Ittiop. 2: 61-64.

AMATO, J.F.R.; W.A. BOEGER \& S.B. AMATO. 1991. Protocolos para laboratório. Coleta e processamento de parasitos do pescado. Rio de Janeiro, 56p.

ANDERSON, D.P. 1990. "Inmunological Indicators: Effects of environmental stress on inmune protection and disease outbreaks". Am. Fish. Society Symposium 8: $38-50$.

Boeger, W.A.; W.S. HusaK \& M.L. MARTINS. 1995. Neotropical Monogenoidea. 25. Anacanthorus penilabiatus n.sp. (Dactylogyridae: Anacanthorinae) from Piaractus mesopotamicus Holmberg, 1887, cultivated in the State of São Paulo, Brazil. Mem. Inst. Oswaldo Cruz 90 (6): 699-701.

Buchmann, K.; M. Kole \& P. Prento. 1987. The nutrition of the gill parasitic monogenean Pseudodactylogyrus anguillae. Parasitol. Res. 73: 532-537.

Ceccarelli, P.S.; L.B. Figueira; C.L.B. Ferraz de Lima \& C.A. Oliveira. 1990. Observações sobre a ocorrência de parasitos no CEPTA entre 1983 e 1990. Bol. Téc. CEPTA 3: 43-54.

ChEnG, T.H.C. 1973. General Parasitology. New York, Academic Press, 965p.

CONROY, G.A. 1987. Curso de ampliación de conocimientos sobre ictiopatologia. Tecnicas de fijacion y coloración para el estudio de parasitos de peces. Maracay, FAO/FCV/UCV, 123p.

CUSACK, R. 1986. Development of infections of Gyrodactylus colemanensis Mizelle and Kritsky, 1967 (Monogenea) and the effect on fry of Salmo gairdneri Richardson. J. Parasitol. 75: 663-668.

Duhamel, G.E.; M.L. Kent; N.O. Dybdal \& R.P. Hedrick. 1986. Henneguya exilis Kudo associated with granulomatous branchitis of channel catfish 
Ictalurus punctatus (Rafinesque). Vet. Pathol. 23: 354-361.

DYKOVA, I. \& J. LOM. 1978. Histopathological changes in fish gills infected with myxosporidian parasites of the genus Henneguya. J. Fish Biol. 12: 197-202.

ELLIS, A.E. 1981. Non-specific defense mechanisms in fish and their role in disease processes, p.337-353. In: W. HENNESSEN (Ed.). Developments in Biological

Standardization. S. Korger, Basel, vol. 49.

Ellis, A. E.; L.S. Munroe \& R.J. RoberTs. 1976. "Defence mechanisms in fish". J. Fish Biol. 8: 67-78.

EWING, M.S.; K.M. KoCAN \& S.A. EwING. 1985. Ichthyophthirius multifiliis (Ciliophora) invasion of gill epithelium. J. Protozool. 32 (2): 305-310.

Figueira, L.B. \& P.S. CecCarelli. 1991. Observações sobre a presença de ectoparasitos em pisicculturas tropicais de interior (CEPTA e Região). Bol. Téc. CEPTA 4 (1): 57-65.

Herraez, M.P. \& A.G. Zapata. 1986. "Structure and funtion of MelanoMacrophage Centres of the Goldfish Carassius auratus". Vet. Immunol. Immunopathol. 12: 117-126.

HINES, R.S. \& D.T. SPIRA. 1974. Ichthyophthiriasis in the mirror carp Cyprinus carpio (L). III. Pathology. J. Fish. Biol. 6: 189-196.

Kalavati, C. \& C.C. NARASIMHA-MuRTI. 1985. Histopathological changes in the gills of Channa punctatus Bl. Infected with Henneguya waltairensis. Arch. Protistenk. 129: 199-202.

Kinkelin, P.; C. Michel \& P. Ghittino. 1991. Tratado de las Enfermedades de los Peces. Zaragoza, España, Ed. Acribia, 353p.

KurovsKAYA, L.N. \& S.A. OSADCHAYA. 1993. The influence of Ichthyophthirius multifiliis on underyearling carp, Cyprinus carpio. J. Ichthyol. 33 (4): 81-92.

LEIBOVITZ, L. 1980a. Ichthyophthiriasis. J.A.V.M.A. 176: 30-31.

1980b. Monogenetic trematode infections. J.A.V.M.A. 7: 608-609.

Martins, M.L.; V.N. SouzA; F.R. Moraes; J.R.E. Moraes; A.J. Costa \& U.F. RocHA. (no prelo). Infections of Henneguya sp. Thélohan, 1892 (Myxozoa: Myxobolidae) on captive Piaractus mesopotamicus Holmberg, 1887, in Brazil, with associated pathology and possible behavioral effects. J. World Aquac. Soc.

MCCraren, J.P.; M.L. LANdolt \& G.L. Hoffman. 1975. Variation in response of channel catfish to Henneguya sp. infections (Protozoa: Myxosporidea). J.

Wildl. Dis. 11: 2-7.

Prieto, A.; E. Fajer; M. Vinjoy \& M. Martinez. 1994. Parasites of freshwater cultured fish. Differential diagnostic keys. June, Mexico, FAO, 60p.

Roberts, R.J. 1981. Patologia de los Peces. Mundi Prensa, Madrid, 366p.

RoberTS, R.J. \& A.M. BULlock. 1980. The skin surface ecosystem of teleost fishes. Proc. R. Soc. Edynburg 79 B: 87-91.

Stoskopf, M.K. 1993. Fish medicine. Pensylvania, W.B. Saunders Co., Harcourt Brace Jovanovich Inc., 882p.

Torroela, J.J. 1988. Aspectos generales de patologia infecciosa, p. 1-36. In: J.S. de los Monteros \& U. Labarta (Eds). Patologia en Acuicultura. Madrid, 
Mundi Prensa, 550p.

VENTURA, M.T. \& I. PAPERNA. 1985. Histopathology of Ichthyophthirius multifiliis infections in fishes. J. Fish. Biol. 27: 185-203.

Recebido em 30.XI.1995; aceito em 17.X.1996. 